\title{
On Accuracy Estimation Using Parametric Bootstrap in small Area Prediction Problems
}

\author{
Tomasz $\dot{Z} a d t o{ }^{1}$
}

\begin{abstract}
We consider longitudinal data and the problem of prediction of subpopulation (domain) characteristics that can be written as a linear combination of the variable of interest, including cases of small or zero sample sizes in the domain and time period of interest. We consider the empirical version of the predictor proposed by Royall (1976) showing that it is a generalization of the empirical version of the predictor presented by Henderson (1950). We propose a parametric bootstrap MSE estimator of the predictor. We prove its asymptotic unbiasedness and derive the order of its bias. Considerations are supported by Monte Carlo simulation analyses to compare its accuracy (not only the bias) with other MSE estimators, including jackknife and weighted jackknife MSE estimators that we adapt for the considered predictor.
\end{abstract}

Key words: Empirical best linear unbiased predictor; model approach in survey sampling; parametric bootstrap; properties of MSE estimators; small area estimation.

\section{Introduction}

To estimate or to predict subpopulation characteristics with small or even zero sample sizes, small area estimation methods are used. In the model approach, empirical (estimated) versions of the best linear unbiased predictor (EBLUP) proposed by Henderson (1950) are widely studied. The first approximation (based on the Taylor's expansion) of the MSE of the predictor is proposed by Kackar and Harville (1984), but they do not study the order of the approximation and the order of the bias of their MSE estimator. These problems are discussed by Prasad and Rao (1990), although in cases where estimators of model parameters are unbiased. Datta and Lahiri (2000) generalize the idea for biased estimators of model parameters including maximum likelihood and restricted maximum likelihood estimators as special cases. More general model and different assumptions leading to different asymptotic results are studied by Das et al. (2004). In all of the above mentioned papers, the additional term of the MSE, resulting from the estimation of model parameters, is derived based on the Taylor's expansion. However, alternatively the jackknife method, proposed by Jiang et al. (2002), or the weighted jackknife method, see Chen and Lahiri (2002, 2003), can be used as well. A similar solution, but using the parametric bootstrap method, is presented in Butar and Lahiri (2003). The parametric bootstrap MSE estimator can also be defined in a different

${ }^{1}$ University of Economics in Katowice, Department of Statistics, Econometrics and Mathematics, 50, 1 Maja Street, 40-287 Katowice, Poland. Email: tomasz.zadlo@ue.katowice.pl 
way and under more general models as shown by González-Manteiga et al. (2007), and González-Manteiga et al. (2007, 2008).

However, the empirical version of the best linear unbiased predictor proposed by Henderson (1950) is not the predictor of the linear combination of the variable of interest (that we would like to predict). This predictor can also be used to make predictions for the unsampled part of the population, as described for example, in Rao and Molina (2015, 7.1.3). Even in this case, they are not generally the best linear unbiased predictors of the linear combination of the variable of interest. The additional condition is required, as we show in Section 3.

In this case, as well as under some additional assumptions presented in Section 3, the resulting predictor becomes the empirical best linear unbiased predictor (EBLUP) of the linear combination of the variable of interest.

A more general predictor is the empirical version of the best linear unbiased predictor proposed by Royall (1976), but it is very rarely considered in the small area estimation literature, for example, Rao and Molina $(2015,178)$ only mentioned it once. We compare it analytically with the empirical version of the best linear unbiased predictor proposed by Henderson (1950). The aim of the article is to propose an asymptotically unbiased parametric bootstrap MSE estimator of the predictor and derive the order of its bias, generalizing the results of Butar and Lahiri (2003) for:

- a more general predictor (see Section 3 and Remark 1 in Section 4),

- and a more general model, taking into account the problem of changes of domain affiliations of population elements and covering many models considered in small area estimation.

Furthermore, our proposal of the MSE estimator will have the advantage that the additional component of the MSE resulting from the estimation of model parameters will not have to be derived, unlike in the case of Taylor's expansion, considered, for example, by Żądło (2009).

In Section 2, we will present our proposal of the superpopulation model, which is a longitudinal linear mixed model with the block-diagonal covariance matrix. It covers many models known from small area estimation literature and allows taking into account possible changes of population and subpopulations in time. In Section 3, we will show that the BLUP proposed by Royall (1976) is a generalization of the BLUP proposed by Henderson (1950) together with a condition when they are equivalent. The dependence between their MSE components will be also presented. In Section 4, we will study the empirical version of the BLUP proposed by Royall (1976) and propose an estimator of its MSE based on the parametric bootstrap method. We will also prove that the order of the bias of the proposed bootstrap MSE estimator is $o\left(K^{-1}\right)$, where $K$ is the number of blocks in the covariance matrix of the variable of interest. Considerations are supported by simulation studies based on real data.

\section{Longitudinal Superpopulation Model}

We will introduce a superpopulation model which belongs to the class of linear mixed models with a block-diagonal variance-covariance matrix of the variable of interest. 
For further considerations the diagonal structure will be crucial, but we will also show that our model is (in our opinion) very flexible in the sense that it covers different types of longitudinal data even in cases of population and subpopulation changes in time. We will also present many longitudinal models used for small area estimation purposes as special cases of our proposal. In the next two sections, different predictors and their MSEs will be studied under the model.

Longitudinal data for periods $t=1, \ldots, M$ are considered, where the number $M$ may include future periods. In the period $t$ the population of size $N_{t}$ is denoted by $\Omega_{t}$. The population in the period $t$ is divided into $D$ disjoint subpopulations (domains) $\Omega_{d t}$ of size $N_{d t}$, where $d=1, \ldots, D$. Let the set of population elements for which observations are available in the period $t$ be denoted by $s_{t}$ and its size by $n_{t}$. The set of subpopulation elements for which observations are available in the period $t$ is denoted by $s_{d t}$ and its size by $n_{d t}$. Let $\Omega_{r d t}=\Omega_{d t} \backslash s_{d t}, N_{r d t}=N_{d t}-n_{d t}, \cup_{t=1}^{M} \Omega_{t}=\Omega, \overline{\bar{\Omega}}=N, \cup_{t=1}^{M} \Omega_{d t}=\Omega_{d}$, $\overline{\bar{\Omega}}_{d}=N_{d}, \cup_{t=1}^{M} \Omega_{r d t}=\Omega_{r d}, \bar{\Omega}_{r d}=N_{r d}, \cup_{t=1}^{M} s_{t}=s, \overline{\bar{s}}=n, \cup_{t=1}^{M} s_{d t}=s_{d}, \overline{\bar{s}}_{d}=n_{d}$, If the period $t$ is the future period, then $s_{t}=s_{d t}=\emptyset, n_{t}=n_{d t}=0, \Omega_{r d t}=\Omega_{d t}$ and $N_{r d t}=N_{d t}$.

Let the vector of random variables of interest in $M$ periods, denoted by $\mathbf{Y}$, be divided into $K$ subvectors denoted by $\mathbf{Y}_{k}$, where $k=1,2, \ldots, K$. The division can be made according to any rule, including but not limited to the division based on subsets $\Omega_{t}(t=1, \ldots, M$ and hence $K=M), \Omega_{d}(d=1, \ldots, D$ and hence $K=D)$ or $\Omega_{d t}(t=1, \ldots, M, d=1,2, \ldots, D$ and hence $K=D \times M)$. We assume that population data obey the following model:

$$
\left\{\begin{array}{c}
\mathbf{Y}_{k}=\mathbf{X}_{k} \beta+\mathbf{Z}_{k} \mathbf{v}_{k}+\mathbf{e}_{k} \\
E\left(\mathbf{e}_{k}\right)=0 \\
E\left(\mathbf{v}_{k}\right)=0 \\
D^{2}\left[\begin{array}{c}
\mathbf{v}_{k} \\
\mathbf{e}_{k}
\end{array}\right]=\left[\begin{array}{cc}
\mathbf{G}_{k}(\boldsymbol{\delta}) & 0 \\
0 & \mathbf{R}_{k}(\boldsymbol{\delta})
\end{array}\right]
\end{array}\right.
$$

where $\mathbf{Y}_{k}$ of size $N_{k} \times 1$ (where $k=1,2, \ldots, K$ ) are assumed to be independent, $\mathbf{X}_{k}$ and $\mathbf{Z}_{k}$ are known matrices of auxiliary variables of sizes $N_{k} \times p$ and $N_{k} \times r_{k}$, respectively, and of full ranks, $\boldsymbol{\delta}$ is a vector of $q$ unknown parameters, $\beta$ is a vector of $p$ unknown parameters, random vectors $\mathbf{e}_{k}$ and $\mathbf{v}_{k}$ of sizes $N_{k} \times 1$ and $r_{k} \times 1$, respectively, are independent and the symbol $D^{2}$ (.) denotes the variance-covariance matrix. Hence, the covariance matrix of $\mathbf{Y}_{k}$ denoted by $D^{2}\left(\mathbf{Y}_{k}\right)=\mathbf{V}_{k}(\boldsymbol{\delta})$ is given by:

$$
\mathbf{V}_{k}(\boldsymbol{\delta})=\mathbf{Z}_{k} \mathbf{G}_{k}(\boldsymbol{\delta}) \mathbf{Z}_{k}^{T}+\mathbf{R}_{k}(\boldsymbol{\delta}) .
$$

If we assume, without loss of generality, that first $n_{k}$ elements of $\mathbf{Y}_{k}$ are observed then matrices in Equation (1) can be decomposed as follows: where $\mathbf{Y}_{k}=\left[\begin{array}{ll}\mathbf{Y}_{s k}^{T} & \mathbf{Y}_{r k}^{T}\end{array}\right]^{T}, \mathbf{X}_{k}=$ $\left[\begin{array}{ll}\mathbf{X}_{s k}^{T} & \mathbf{X}_{r k}^{T}\end{array}\right]^{T}, \mathbf{Z}_{k}=\left[\begin{array}{ll}\mathbf{Z}_{s k}^{T} & \mathbf{Z}_{r k}^{T}\end{array}\right]^{T}, \mathbf{e}_{k}=\left[\begin{array}{ll}\mathbf{e}_{s k}^{T} & \mathbf{e}_{r k}^{T}\end{array}\right]^{T}, \mathbf{Y}_{s k}, \mathbf{Y}_{r k}, \mathbf{X}_{s k}, \mathbf{X}_{r k}, \mathbf{Z}_{s k}, \mathbf{Z}_{r k}, \mathbf{e}_{s k}, \mathbf{e}_{r k}$ are of sizes $n_{k} \times 1,\left(N_{k}-n_{k}\right) \times 1, n_{k} \times p,\left(N_{k}-n_{k}\right) \times p, n_{k} \times r_{k},\left(N_{k}-n_{k}\right) \times r_{k}, n_{k} \times$ $1,\left(N_{k}-n_{k}\right) \times 1$, respectively. In this case, covariance matrices in Equation (2) are given by: $\mathbf{V}_{k}=\left[\begin{array}{ll}\mathbf{V}_{k s s} & \mathbf{V}_{k s r} \\ \mathbf{V}_{k r s} & \mathbf{V}_{k r r}\end{array}\right]$ and $\mathbf{R}_{k}=\left[\begin{array}{ll}\mathbf{R}_{k s s} & \mathbf{R}_{k s r} \\ \mathbf{R}_{k r s} & \mathbf{R}_{k r r}\end{array}\right]$, where matrices $\mathbf{V}_{k s s}$ and $\mathbf{R}_{k s s}$ are $n_{k} \times n_{k}, \mathbf{V}_{k r r}$ and $\mathbf{R}_{k r r}$ are $\left(N_{k}-n_{k}\right) \times\left(N_{k}-n_{k}\right), \mathbf{V}_{k s r}$ and $\mathbf{R}_{k s r}$ are $n_{k} \times\left(N_{k}-n_{k}\right), \mathbf{V}_{k r s}=$ $\mathbf{V}_{k s r}^{T}$ and $\mathbf{R}_{k r s}=\mathbf{R}_{k s r}^{T}$. 
Model (1) can be written as follows:

$$
\mathbf{Y}=\mathbf{X} \beta+\mathbf{Z v}+\mathbf{e},
$$

where $\mathbf{Y}=\operatorname{col}_{1 \leq k \leq K}\left(\mathbf{Y}_{k}\right), \quad \mathbf{X}=\operatorname{col}_{1 \leq k \leq K}\left(\mathbf{X}_{k}\right), \quad \mathbf{Z}=\operatorname{diag}_{1 \leq k \leq K}\left(\mathbf{Z}_{k}\right), \quad \mathbf{v}=\operatorname{col}_{1 \leq k \leq K}\left(\mathbf{v}_{k}\right)$, $\mathbf{e}=\operatorname{col}_{1 \leq k \leq K}\left(\mathbf{e}_{k}\right)$. Hence, the covariance matrix of $\mathbf{Y}$ denoted by $D^{2}(\mathbf{Y})=\mathbf{V}(\boldsymbol{\delta})$ is given by:

$$
\mathbf{V}(\boldsymbol{\delta})=\mathbf{Z G}(\boldsymbol{\delta}) \mathbf{Z}^{T}+\mathbf{R}(\boldsymbol{\delta}),
$$

where $\mathbf{G}=\operatorname{diag}_{1 \leq k \leq K}\left(\mathbf{G}_{k}\right)$ and $\mathbf{R}=\operatorname{diag}_{1 \leq k \leq K}\left(\mathbf{R}_{k}\right)$.

Model (1) is a very general model covering many special cases, including cases presented below, in which changes of the population and subpopulations are taken into account. The assumption that one population element may change its domain (or group) affiliation in time is very important in longitudinal surveys. For example, let us consider the population of households and the division of the population into domains made according to household size. In this case, we should assume that some households can change their sizes in time and hence their domain affiliation. If some human population is under the study, one may be interested in its characteristics for subpopulations defined according to some social or economic criteria (e.g., the job position). In the case of business surveys, the population of firms may be divided into subpopulations according to some economic or financial criteria, which may imply even stronger changes in the division of the population in time than in the case of human populations.

Let $M_{i d}$ denotes the number of periods when the $i$ th population element belongs to the $d$ th domain (it may include future periods). Values of the variable of interest are realizations of random variables $Y_{i d j}$ for the $i$ th population element that belongs to the $d$ th domain in the period $t_{i j}$, where $i=1,2, \ldots, N, j=1,2, \ldots, M_{i d}, d=1,2, \ldots, D$. The vector $\mathbf{Y}_{i d}=\left[Y_{i d j}\right]_{M_{i d}} \times 1$ is called the profile. We have defined $\Omega=\cup_{t=1}^{M} \Omega_{t}$, where $\Omega_{t}$ is the population in the period $t$. Now, let us assume that profiles in $\Omega$ are divided into $K$ groups $\Omega_{k}$ for which where the number of random variables within each group equals $N_{k}$, where $k=1,2, \ldots, K$. Grouping profiles means that groups always have a longitudinal character in this special case of Model (1). We consider the following four cases:

- $\mathrm{C} 1$ - the additional division of the profiles is not taken into account and hence, the number of groups of profiles equals the number of profiles $(K=N)$,

- C2 - domains $\Omega_{d}(d=1,2, \ldots, D)$ are unions of groups of profiles $\Omega_{k}(K>D$ and $K \neq N$ ),

- C3 - groups of profiles are domains $\Omega_{d}(d=1,2, \ldots, D)$ and hence $K=D$,

- C4 - sets $\Omega_{k}(k=1,2, \ldots, K)$ are unions of domains $\Omega_{d}(K<D)$.

Now we can define the profile more precisely, including the information on the division of the population into $K$ groups of profiles. The vector of random variables for the $i$ th population element in different periods will be denoted by $\mathbf{Y}_{i d k}=\left[Y_{i d j k}\right]_{M_{i d k} \times 1}$, where $M_{i d k}$ is the number of periods when the $i$ th element belongs to the $d$ th domain and the $k$ th group of profiles. Let the number of profiles within the $k$ th group be denoted by $M_{k}$. Hence, the number of random variables within $\Omega_{k}$ equals $N_{k}=\sum_{i=1}^{M_{k}} M_{i d k}$. In the cases C1 and C3, the additional subscript $k$ is not necessary because $i$ and $d$ explicitly define $k$. Let $m_{i d k}$ be the number of periods when the $i$ th population element (which belongs to the $d$ th domain 
and the $k$ th group of profiles) is observed. The vector $\mathbf{Y}_{s i d k}=\left[Y_{i d k j}\right]_{m_{i d k} \times 1}$, will be called the sample profile. Let the number of profiles observed in the sample in the $k$ th group be denoted by $m_{k}$. Hence, the number of sample observations from $\Omega_{k}$ equals $n_{k}=\sum_{i=1}^{m_{k}} m_{i d k}$. Let the vector $\mathbf{Y}_{\text {ridk }}=\left[Y_{i d k j}\right]_{M_{\text {ridk }} \times 1}$, where $M_{\text {ridk }}=M_{i d k}-m_{i d k}$, be the profile of random variables with non-observed realizations (including out of sample and future values). This notation allows the inclusion of possibilities of changes of the population in time, changes of subpopulations in time and even changes of domains or group affiliations of population elements in time in the considerations.

Let us consider the cases in which random variables for one population element observed in different periods form more than one profile. It is possible in the following cases:

- for $\mathrm{C} 1$ : a change of the domain affiliation,

- for $\mathrm{C} 2$ : a change of the group affiliation within a domain or a change of the domain affiliation,

- for C3: a change of the domain affiliation equivalent to a change of the group affiliation,

- for $\mathrm{C} 4$ : the change of the domain affiliation within the group or the change of the group and the domain affiliation at the same time.

It must be pointed out that the period of time can be denoted by one out of two indexes: $t$ (where $t=1,2, \ldots, M$ ) - to distinguish between different periods of the longitudinal data, and $j$ (where $j=1,2, \ldots, M_{i d}$ ) - to distinguish between different periods within profiles.

Model (1) covers many unit-level longitudinal models with block-diagonal covariance matrix considered in the literature. Case $\mathrm{C} 1$ of the model includes, for example, the model with independent profile specific random effects considered by Verbeke and Molenbergh (2000, 20). Random regression coefficient models considered by Hobza and Morales (2013) and the multilevel model studied by Moura and Holt (1999), both assumed for one period, and also heteroscedastic models with domain specific and domain and domain-and-time specific random effects considered by Morales and Santamaría (2019) are special cases of case C3. The model with two random effects (domain specific and profile specific) considered by Stukel and Rao (1999) and Nissinen $(2009,22)$ and the model with two random effects (domain specific and domain-and-time specific) considered by Molina et al. (2010, 143-180) are covered by the case C3 of our model as well. Case C3 also covers models considered by Żądło (2014, 2015a) with profile-specific random effects spatially correlated within domains. Case $\mathrm{C} 4$ of the model includes the model with profile-specific random effects spatially correlated within groups of domains proposed by Żądło (2015b). What is more, longitudinal area-level models considered by Rao and Yu (1994) and Marhuenda et al. (2013) can also be written as a Case C3 of our model. However, not all special cases of Model (1) are covered by Cases $\mathrm{C} 1-\mathrm{C} 4$ due to assumed non-longitudinal character of groups including the longitudinal model with domain-and-time specific random effects (autocorrelated in time) considered by Saei and Chambers $(2003,13)$.

It must be pointed out that Model (1) does not cover linear mixed models without blockdiagonal covariance matrix, see models studied by, for example Fabrizi et al. (2007, 189), Pagliarell and Salvatore (2016, 232-235), D’Aló et al. (2017), and nonlinear mixed 
models studied by, for example Hobza et al. (2018). The overview of different small area models can be found in Jiang and Lahiri (2006).

\section{Best Linear Unbiased Predictors}

Under the linear mixed model presented in the previous section, we will compare analytically different predictors and their MSEs, including a predictor that is widely discussed in the literature, proposed by Henderson (1950) and a more general predictor presented by Royall (1976).

We study the problem of prediction of a linear combination $\theta=\gamma^{T} \mathbf{Y}$. For example, if we are interested in the prediction of the $d$ th subpopulation (domain) total in the period $t$ then the $k$ th element of the vector $\gamma$ equals 1 for $k \in \Omega_{d t}$ and 0 otherwise. Under Model (3):

$$
\theta=\gamma^{T} \mathbf{Y}=\gamma^{T} \mathbf{X} \beta+\gamma^{T} \mathbf{Z v}+\gamma^{T} \mathbf{e} .
$$

Let us present the results obtained by Henderson (1950) for surveys conducted in one period by changing the sizes of matrices in his model to cover the case of the longitudinal Model (1). He considers the problem of prediction of:

$$
\theta^{s}=\mathbf{l}^{T} \beta+\mathbf{m}^{T} \mathbf{v}
$$

For $\mathbf{l}^{T}=\gamma^{T} \mathbf{X}$ and $\mathbf{m}^{T}=\gamma^{T} \mathbf{Z}$ from Equations (5a) and (5b) we obtain:

$$
\theta=\theta^{s}+\gamma^{T} \mathbf{e}
$$

Theorem 1. (see Henderson 1950). Assume that sample data obey the following assumptions:

$$
\left\{\begin{array}{c}
\mathbf{Y}_{s}=\mathbf{X}_{s} \beta+\mathbf{Z} \mathbf{v}+\mathbf{e}_{s} \\
E\left(\mathbf{e}_{s}\right)=0 \\
E(\mathbf{v})=0 \\
D^{2}\left[\begin{array}{c}
\mathbf{v} \\
\mathbf{e}_{s}
\end{array}\right]=\left[\begin{array}{cc}
\mathbf{G}(\boldsymbol{\delta}) & 0 \\
0 & \mathbf{R}_{s s}(\boldsymbol{\delta})
\end{array}\right]
\end{array}\right.
$$

Among linear, model-unbiased predictors $\hat{\theta}^{s}=\mathrm{a}^{T} \mathbf{Y}_{s}+\mathbf{b}$ of linear combination of $\beta$ and the realization of $\mathbf{v}$ given by $\theta^{s}=\mathbf{l}^{T} \beta+\mathbf{m}^{T} \mathbf{v}$ (for specified vectors, $\mathbf{l}$ and $\mathbf{m}$, of constants) the MSE is minimized by:

$$
\hat{\theta}_{B L U P}^{s}=\mathbf{1}^{T} \tilde{\beta}(\boldsymbol{\delta})+\mathbf{m}^{T} \tilde{\mathbf{v}}(\boldsymbol{\delta}),
$$

where

$$
\begin{gathered}
\tilde{\beta}(\boldsymbol{\delta})=\left(\mathbf{X}_{s}^{T} \mathbf{V}_{s s}^{-1}(\boldsymbol{\delta}) \mathbf{X}_{s}\right)^{-1} \mathbf{X}_{s}^{T} \mathbf{V}_{s s}^{-1}(\boldsymbol{\delta}) \mathbf{Y}_{s} \\
\tilde{\mathbf{v}}(\boldsymbol{\delta})=\mathbf{G}(\boldsymbol{\delta}) \mathbf{Z}_{s}^{T} \mathbf{V}_{s s}^{-1}(\boldsymbol{\delta})\left(\mathbf{Y}_{s}-\mathbf{X}_{s} \tilde{\beta}(\boldsymbol{\delta})\right)
\end{gathered}
$$


The MSE of $\hat{\theta}_{B L U P}^{s}$ is given by

$$
\operatorname{MSE}\left(\hat{\theta}_{B L U P}^{s}\right)=\operatorname{Var}\left(\hat{\theta}_{B L U P}^{s}-\theta^{s}\right)=g_{1}^{s}(\boldsymbol{\delta})+g_{2}^{s}(\boldsymbol{\delta}),
$$

where

$$
\begin{gathered}
g_{1}^{s}(\boldsymbol{\delta})=\mathbf{m}^{T}\left(\mathbf{G}(\boldsymbol{\delta})-\mathbf{G}(\boldsymbol{\delta}) \mathbf{Z}_{s}^{T} \mathbf{V}_{s s}^{-1}(\boldsymbol{\delta}) \mathbf{Z}_{\mathrm{s}} \mathbf{G}(\boldsymbol{\delta})\right) \mathbf{m} \\
g_{1}^{s}(\boldsymbol{\delta})=\left(\mathbf{1}^{T}-\mathbf{m}^{T} \mathbf{G}(\boldsymbol{\delta}) \mathbf{Z}_{s}^{T} \mathbf{V}_{s s}^{-1}(\boldsymbol{\delta}) \mathbf{X}_{s}\right)\left(\mathbf{X}_{s}^{T} \mathbf{V}_{s s}^{-1}(\boldsymbol{\delta}) \mathbf{X}_{s}\right)^{-1} \times \\
\times\left(\mathbf{1}^{T}-\mathbf{m}^{T} \mathbf{G}(\boldsymbol{\delta}) \mathbf{Z}_{s}^{T} \mathbf{V}_{s s}^{-1}(\boldsymbol{\delta}) \mathbf{X}_{s}\right)^{T}
\end{gathered}
$$

The proof of the theorem (for surveys conducted in one period) is presented in detail in, for example, Rao and Molina (2015, 119-120).

Because in Theorem 1 the problem of prediction of Equation (5b) instead of Equation (5c) is considered, in small area estimation literature, see, for example, Rao and Molina $(2015,178-179)$, the problem of prediction of Equation $(5 \mathrm{c})$ using predictor $(7 \mathrm{a})$ is studied. We assume longitudinal Model (1) and study the problem of prediction of Equation (5c), but in a more general framework than in Rao and Molina (2015, 178-179), where the crucial difference is the lack of the independence assumption of random components.

Let us consider the problem of prediction of

$$
\theta=\gamma^{T} \mathbf{Y}=\gamma_{s}^{T} \mathbf{Y}_{s}+\gamma_{r}^{T} \mathbf{Y}_{r}=\gamma_{s}^{T} \mathbf{Y}_{s}+\theta_{r}
$$

where $\gamma=\left[\gamma_{s}^{T} \gamma_{r}^{T}\right]^{T}, \theta_{r}=\gamma_{r}^{T} \mathrm{Y}_{r}$ and the realization of $\gamma_{s}^{T} \mathrm{Y}_{s}$ is known. Because realization of $\gamma_{s}^{T} \mathrm{Y}_{s}$ is known, the problem of prediction of Equation (8) is reduced to the problem of prediction of:

$$
\theta_{r}=\gamma_{r}^{T} \mathbf{Y}_{r}=\gamma_{r}^{T} \mathbf{X}_{r} \beta+\gamma_{r}^{T} \mathbf{Z}_{r} \mathbf{v}+\gamma_{r}^{T} \mathbf{e}_{r}=\theta_{r}^{s}+\gamma_{r}^{T} \mathbf{e}_{r}
$$

where

$$
\theta_{r}^{s}=\gamma_{r}^{T} \mathbf{X}_{r} \beta+\gamma_{r}^{T} \mathbf{Z}_{r} \mathbf{v}
$$

Based on Theorem 1 the BLUP of Equation (10) is given by:

$$
\hat{\theta}_{B L U P r}^{s}=\mathbf{1}^{T} \tilde{\beta}(\boldsymbol{\delta})+\mathbf{m}^{T} \tilde{\mathbf{v}}(\boldsymbol{\delta}),
$$

where $\mathbf{1}^{T}=\gamma_{r}^{T} \mathbf{X}_{r}, \mathbf{m}^{T}=\gamma_{r}^{T} \mathbf{Z}_{r}, \tilde{\beta}(\boldsymbol{\delta})$ and $\tilde{\mathbf{v}}(\boldsymbol{\delta})$ are given by Equations (7b) and (7c), respectively.

Finally, we obtain the following predictor:

$$
\begin{aligned}
& \hat{\theta}=\gamma_{s}^{T} \mathbf{Y}_{s}+\hat{\theta}_{B L U P r}^{s}=\gamma_{s}^{T} \mathbf{Y}_{s}+ \\
& +\gamma_{r}^{T}\left[\mathbf{X}_{r} \tilde{\beta}(\boldsymbol{\delta})+\left(\mathbf{Z}_{r} \mathbf{G}(\boldsymbol{\delta}) \mathbf{Z}_{s}^{T}\right) \mathbf{V}_{s s}^{-1}(\boldsymbol{\delta})\left(\mathbf{Y}_{s}-\mathbf{X}_{s} \tilde{\beta}(\boldsymbol{\delta})\right)\right]
\end{aligned}
$$

of Equation (8), which generally is not the BLUP of Equation (8). Later in this section, we will show that if $\mathbf{R}_{s r}=0$, then the predictor given by Equation (12a) becomes the BLUP of Equation (8). 
The MSE of the predictor (12a) of Equation (8) is given by

$$
\begin{aligned}
& \operatorname{MSE}(\hat{\theta})=E\left(\gamma_{s}^{T} \mathbf{Y}_{s}+\hat{\theta}_{B L U P r}^{s}-\gamma^{T} \mathbf{Y}\right)^{2}= \\
& =E\left(\hat{\theta}_{B L U P r}^{s}-\gamma_{r}^{T} \mathbf{Y}_{r}\right)^{2}= \\
& =E\left(\hat{\theta}_{B L U P r}^{s}-\theta_{r}^{s}-\gamma_{r}^{T} \mathbf{e}_{r}\right)^{2}= \\
& =\operatorname{MSE}\left(\hat{\theta}_{B L U P r}^{s}\right)+ \\
& \quad+g_{4}^{s}(\boldsymbol{\delta})-2 \operatorname{Cov}\left(\left(\hat{\theta}_{B L U P r}^{s}-\theta_{r}^{s}\right), \gamma_{r}^{T} \mathbf{e}_{r}\right),
\end{aligned}
$$

where $\operatorname{MSE}\left(\hat{\theta}_{B L U P r}^{S}\right)$ is given by Equation (7d) (where $\mathbf{1}^{T}=\gamma_{r}^{T} \mathbf{X}_{r}$ and $\mathbf{m}^{T}=\gamma_{r}^{T} \mathbf{Z}_{r}$ ), $g_{4}^{S}(\boldsymbol{\delta})=\gamma_{r}^{T} \mathbf{R}_{r r}(\delta) \gamma_{r}$ and

$$
\begin{aligned}
\operatorname{Cov} & \left(\left(\hat{\theta}_{B L U P r}^{s}-\theta_{r}^{s}\right), \gamma_{r}^{T} \mathbf{e}_{r}\right)= \\
& =\gamma_{r}^{T} \mathbf{X}_{r}\left(\mathbf{X}_{s}^{T} \mathbf{V}_{s s}^{-1}(\boldsymbol{\delta}) \mathbf{X}_{s}\right)^{-1} \mathbf{X}_{s}^{T} \mathbf{V}_{s s}^{-1} \mathbf{R}_{s t}(\boldsymbol{\delta}) \gamma_{r}+ \\
& +\gamma_{r}^{T} \mathbf{Z}_{r} \mathbf{G}(\boldsymbol{\delta}) \mathbf{Z}_{s}^{T} \mathbf{V}_{s s}^{-1}(\boldsymbol{\delta}) \times \\
& \times\left(\mathrm{I}-\mathbf{X}_{s}\left(\mathbf{X}_{s}^{T} \mathbf{V}_{s s}^{-1}(\boldsymbol{\delta}) \mathbf{X}_{s}\right)^{-1} \mathbf{X}_{s}^{T} \mathbf{V}_{s s}^{-1}(\boldsymbol{\delta})\right) \mathbf{R}_{s r}(\boldsymbol{\delta}) \gamma_{r}
\end{aligned}
$$

Firstly, we assume that $\mathrm{R}_{s r}=0$. Then, $\operatorname{Cov}\left(\left(\hat{\theta}_{B L U P r}^{s}-\theta_{r}^{s}\right), \gamma_{r}^{T} \mathbf{e}_{r}\right)=0$ and Equation (12b) simplifies to

$$
\begin{gathered}
\operatorname{MSE}(\hat{\theta})=\operatorname{MSE}\left(\hat{\theta}_{B L U P r}^{s}\right)+g_{4}^{s}(\boldsymbol{\delta})= \\
=g_{1}^{s}(\boldsymbol{\delta})+g_{2}^{s}(\boldsymbol{\delta})+g_{4}^{s}(\boldsymbol{\delta})
\end{gathered}
$$

and the predictor (12a) is the BLUP of Equation (8) (which will be shown later in this section).

Royall (1976) for surveys conducted in one period derived a more general predictor than predictors (7a) and (12a). In the following consideration we change the sizes of matrices in his theorem to cover the following longitudinal model:

$$
\left\{\begin{array}{l}
E(\mathbf{Y})=\mathbf{X} \beta \\
D^{2}(\mathbf{Y})=\mathbf{V}
\end{array}\right.
$$

where matrices in Equation (13) were defined in Section 1.

Theorem 2. (compare Royall 1976). Assume that the population data obey the longitudinal general linear model (see Equation (13)). Among linear, model-unbiased predictors $\hat{\theta}=g^{T} \mathbf{Y}_{s}$ of linear combination of random variables $\theta=\gamma^{T} \mathbf{Y}$, the MSE is 
minimized by:

$$
\hat{\theta}_{B L U P}=\gamma_{s}^{T} \mathbf{Y}_{s}+\gamma_{r}^{T}\left[\mathbf{X}_{r} \tilde{\beta}(\boldsymbol{\delta})+\mathbf{V}_{r s}(\boldsymbol{\delta}) \mathbf{V}_{s s}^{-1}(\boldsymbol{\delta})\left(\mathbf{Y}_{s}-\mathbf{X}_{s} \tilde{\beta}(\boldsymbol{\delta})\right)\right]
$$

where $\tilde{\boldsymbol{\beta}}(\boldsymbol{\delta})$ is given by (7b).

The MSE of $\hat{\theta}_{B L U P}$ is given by

$$
\operatorname{MSE}\left(\hat{\theta}_{B L U P}\right)=g_{1}(\boldsymbol{\delta})+g_{2}(\boldsymbol{\delta}),
$$

where

$$
\begin{gathered}
g_{1}(\boldsymbol{\delta})=\gamma_{r}^{T}\left(\mathbf{V}_{r r}(\boldsymbol{\delta})-\mathbf{V}_{r s}(\boldsymbol{\delta}) \mathbf{V}_{s s}^{-1}(\boldsymbol{\delta}) \mathbf{V}_{s r}(\boldsymbol{\delta})\right) \gamma_{r}, \\
g_{2}(\boldsymbol{\delta})=\gamma_{r}^{T}\left(\mathbf{X}_{r}-\mathbf{V}_{r s}(\boldsymbol{\delta}) \mathbf{V}_{s s}^{-1}(\boldsymbol{\delta}) \mathbf{X}_{s}\right) \\
\times\left(\mathbf{X}_{s}^{T} \mathbf{V}_{s s}^{-1}(\boldsymbol{\delta}) \mathbf{X}_{s}\right)^{-1} \times\left(\mathbf{X}_{r}-\mathbf{V}_{r s}(\boldsymbol{\delta}) \mathbf{V}_{\mathrm{ss}}^{-1}(\boldsymbol{\delta}) \mathbf{X}_{s}\right)^{\mathrm{T}} \gamma_{r}
\end{gathered}
$$

The proof of the theorem is presented in details by, for example, Valliant et al. (2000, 29-30).

Firstly, let us compare predictor (14a) proposed by Royall (1976) with predictor (7a) proposed by Henderson (1950). Predictor (14a) can be treated as a generalization of the predictor (7a) because (i) the general linear Model (13) covers the assumptions of the general linear mixed Model (6), (ii) the predictor (14a) is the predictor of $\theta$, while the predictor (7a) is the predictor of $\theta^{s}$ (where (5c) holds) and (iii) $\hat{\theta}^{s}=\mathrm{a}^{T} \mathrm{Y}_{s}+b$ considered in Theorem 1 is of the same form as $\hat{\theta}=\mathrm{g}^{T} \mathrm{Y}_{\mathrm{s}}$ considered in Theorem 2, because $b=0$ under unbiasedness of the predictor.

Secondly, let us compare predictors of $\theta$ given by Equation (14a), proposed by Royall (1976), and given by Equation (12a). Although (14a) is derived under the general linear Model (13), we consider its formula under the special case of the model - the general linear mixed Model (1). Hence, the predictor (14a) simplifies to:

$$
\begin{aligned}
& \hat{\theta}_{B L U P}=\gamma_{s}^{T} \mathbf{Y}_{s}+\gamma_{r}^{T} \mathbf{X}_{r} \tilde{\beta}(\boldsymbol{\delta})+ \\
& +\gamma_{r}^{T}\left(\mathbf{R}_{r s}(\boldsymbol{\delta})+\mathbf{Z}_{r} \mathbf{G}(\boldsymbol{\delta}) \mathbf{Z}_{s}^{T}\right) \mathbf{V}_{s s}^{-1}(\boldsymbol{\delta})\left(\mathbf{Y}_{s}-\mathbf{X}_{s} \tilde{\beta}(\boldsymbol{\delta})\right) .
\end{aligned}
$$

If $\mathbf{R}_{r s}=0$ then predictors (15) are (12a) identical and their MSEs (given by Equations (14b) and (12d), respectively) are equal. Being more precise, if $\mathbf{R}_{r s}=0$, then

$$
g_{1}(\boldsymbol{\delta})=g_{1}^{s}(\boldsymbol{\delta})+g_{4}^{s}(\boldsymbol{\delta})
$$

and

$$
g_{2}(\boldsymbol{\delta})=g_{2}^{s}(\boldsymbol{\delta})
$$

To sum up, the predictor (12a) is the BLUP of $\theta$ only for models where $\mathbf{R}_{r s}=0$. 


\section{Empirical Best Linear Unbiased Predictors}

In the previous section, we assumed that model parameters are known. Now we will take into account additional variability resulting from their estimation and its influence on the MSE and MSE estimation.

Let us start our considerations from the predictor proposed by Henderson (1950). The Best Linear Unbiased Predictor (BLUP) (7a) depends on the variance parameters $\boldsymbol{\delta}$ that are unknown in practical applications. Replacing $\boldsymbol{\delta}$ by an estimator $\hat{\boldsymbol{\delta}}$, we obtain the two-stage predictor $\hat{\theta}_{E B L U P}^{S}=\hat{\theta}_{B L U P}^{S}(\boldsymbol{\delta})$ called empirical (estimated) best linear unbiased predictor EBLUP. In the simulation we will use the Restricted Maximum Likelihood (REML) method, known as robust on nonnormality (Jiang 1996) to estimate $\boldsymbol{\delta}$. Under some weak assumptions presented by Kackar and Harville (1981):

(i) an estimator of the vector of model parameters is any even, translation-invariant estimator, for example the REML estimator,

(ii) the distributions of $\mathbf{v}$ and $\mathbf{e}_{s}$ are both symmetric around 0 ,

(iii) the expectation of the predictor is finite, the EBLUP remains unbiased.

The MSE of EBLUP is greater than the MSE of BLUP due to the estimation of the model parameters. The problem of estimating accuracy of the EBLUP is widely discussed in the literature and results discussed below can be directly used for our case just by changing the sizes of the matrices to cover the longitudinal Model (1). The first paper where the problem of the approximate formula (based on the Taylor expansion) of the MSE of the EBLUP is studied and its estimator is proposed is Kackar and Harville (1984). The authors do not study the orders of the neglected terms in the approximation of the MSE and the order of the bias of the MSE estimator. The pioneering results are presented by Prasad and Rao (1990). They assume, inter alia, block-diagonal variance-covariance matrix, normality of random effects and random components and unbiasedness of $\boldsymbol{\delta}$ which allows obtaining the order of MSE approximation and proving the asymptotically unbiasedness of their MSE estimator. The problem is also studied in detail by Datta and Lahiri (2000), but for a larger class of estimators $\boldsymbol{\delta}$ (including maximum likelihood and restricted maximum likelihood estimators), where unbiasedness is not required. The more general mixed model is studied by Das et al. (2004), who, based on different assumptions obtained different asymptotic results. Jackknife and weighted jackknife estimators of MSE of EBLUP are proposed by Jiang et al. (2002), Chen and Lahiri (2002, 2003). The parametric bootstrap method is used to estimate MSE of EBLUP by, inter alia, Butar and Lahiri (2003), González-Manteiga et al. (2007, 2008) As shown, for example, by Schmid and Münnich (2014), the parametric bootstrap method of MSE estimation is preferable in the case of different predictors as well.

If $\boldsymbol{\delta}$ in the formula of the predictor (14a) proposed by Royall (1976) is replaced by an estimator $\hat{\boldsymbol{\delta}}$, we obtain $\hat{\theta}_{E B L U P}=\hat{\theta}_{B L U P}(\boldsymbol{\delta})$ - the empirical version of the predictor. It remains unbiased under some weak assumptions presented by Żąłło (2004) ((i) and (iii) the same as mentioned above in the case of empirical version of the predictor proposed by Henderson (1950), in assumption (ii) symmetric distribution around zero of e instead of $\mathbf{e}_{s}$ is assumed). For the empirical version of the predictor studied by Royall (1976), the MSE estimator using Taylor's series expansion was proposed by Żądło (2009), who 
generalized the results of Datta and Lahiri (2000) obtained for the empirical version of the predictor considered by Henderson (1950). In this section, we will propose a parametric bootstrap MSE estimator by a generalization of the results presented by Butar and Lahiri (2003). For (i) the more general predictor - we will study the empirical version of the Royall (1976) predictor instead of the empirical version of the Henderson (1950) predictor and (ii) for the more general model (the model proposed in Section 1).

Butar and Lahiri (2003) consider two superpopulation models assumed for sample data. We assume the following superpopulation models, but for the whole population:

- Model 1: Model (1) with an additional assumption of normality of random effects and random components

- Model 2: bootstrap model:

$$
\left\{\begin{array}{c}
\mathbf{Y}_{k}^{*}=\mathbf{X}_{k} \hat{\beta}+\mathbf{Z}_{k} \mathbf{v}_{k}^{*}+\mathbf{e}_{k}^{*} \\
E_{*}\left(\mathbf{e}_{k}^{*}\right)=0 \\
E_{*}\left(\mathbf{v}_{k}^{*}\right)=0 \\
D_{*}^{2}\left[\begin{array}{c}
\mathbf{v}_{k}^{*} \\
\mathbf{e}_{k}^{*}
\end{array}\right]=\left[\begin{array}{cc}
\mathbf{G}_{k}(\hat{\boldsymbol{\delta}}) & 0 \\
0 & \mathbf{R}_{k}(\hat{\boldsymbol{\delta}})
\end{array}\right]
\end{array}\right.
$$

where $\mathbf{Y}_{1}^{*}, \ldots, \mathbf{Y}_{k}^{*}, \ldots, \mathbf{Y}_{K}^{*}$ are independent, $\mathbf{v}_{k}^{*}$ and $\mathbf{e}_{k}^{*}$ are generated from multivariate normal distributions, $\hat{\boldsymbol{\delta}}$ is an estimator of $\boldsymbol{\delta}$ that satisfies the regularity conditions (RC) given in the Appendix (Section 7), $\hat{\beta}=\tilde{\beta}(\hat{\boldsymbol{\delta}})$, where $\tilde{\beta}(\boldsymbol{\delta})$ is given by Equation (7b).

We propose the following parametric bootstrap MSE estimator:

$$
\begin{aligned}
& \widehat{M S E}\left(\hat{\theta}_{E B L U P}\right)=g_{1}(\hat{\boldsymbol{\delta}})+g_{2}(\hat{\boldsymbol{\delta}})+ \\
& -E_{*}\left(g_{1}\left(\boldsymbol{\delta}^{*}\right)+g_{2}\left(\boldsymbol{\delta}^{*}\right)-\left(g_{1}(\hat{\boldsymbol{\delta}})+g_{2}(\hat{\boldsymbol{\delta}})\right)\right)+ \\
& +E_{*}\left(\hat{\theta}_{E B L U P}\left(\mathrm{Y}_{s}, \hat{\beta}\left(\boldsymbol{\delta}^{*}\right), \boldsymbol{\delta}^{*}\right)-\hat{\theta}_{E B L U P}\left(\mathrm{Y}_{s}, \hat{\boldsymbol{\beta}}(\boldsymbol{\delta}), \boldsymbol{\delta}\right)\right)^{2},
\end{aligned}
$$

where $E_{*}$ it is the expectation with respect to Model 2, $\boldsymbol{\delta}^{*}$ is calculated as $\boldsymbol{\delta}$ but based on $\mathbf{Y}_{s}^{*}$ instead of $\mathbf{Y}_{s}$. The formula of the estimator (19) of the empirical version of Royall (1976) predictor is similar to the MSE estimator of the empirical version of Henderson (1950) predictor proposed by Butar and Lahiri (2003). If $g_{1}(),. g_{2}($.$) and \hat{\theta}_{E B L U P}$ in (19) are replaced by $g_{1}^{s}(\cdot), g_{2}^{s}(\cdot)$ and $\hat{\theta}_{E B L U P}^{s}$ respectively, we obtain the Butar and Lahiri (2003) MSE estimator.

We will prove that the estimator (19) is asymptotically unbiased and that the bias is of order $o\left(K^{-1}\right)$. The proof will be a generalization of the proof presented by Butar and Lahiri (2003). Butar and Lahiri (2003) propose another MSE estimator which approximates the parametric bootstrap MSE estimator but its bias, Butar and Lahiri (2003), is of a higher order - the same order as the bias of the naive MSE estimator. 
Lemma 1. Under Model 1 and the regularity conditions (RC) presented in the Appendix (Section 7), we have

$$
\begin{aligned}
& E\left(g_{1}(\boldsymbol{\delta})-B_{\boldsymbol{\delta}}^{T}(\boldsymbol{\delta}) \frac{\partial g_{1}(\boldsymbol{\delta})}{\partial \boldsymbol{\delta}}+g_{3}(\boldsymbol{\delta})\right)=g_{1}(\boldsymbol{\delta})+o\left(K^{-1}\right), \\
& E\left(g_{2}(\boldsymbol{\delta})\right)=g_{2}(\boldsymbol{\delta})+o\left(K^{-1}\right), \\
& E\left(g_{3}(\boldsymbol{\delta})\right)=g_{3}(\boldsymbol{\delta})+o\left(K^{-1}\right),
\end{aligned}
$$

where

$$
g_{3}(\boldsymbol{\delta})=\operatorname{trace}\left(\frac{\partial \mathrm{c}^{\mathrm{T}}(\boldsymbol{\delta})}{\partial \boldsymbol{\delta}} \mathbf{V}_{s s}\left(\frac{\partial \mathrm{c}^{T}(\boldsymbol{\delta})}{\partial \boldsymbol{\delta}}\right)^{T} \Sigma(\boldsymbol{\delta})\right)
$$

and

$$
\mathrm{c}^{T}=\gamma_{r}^{T} \mathbf{V}_{r s} \mathbf{V}_{s s}^{-1}=\gamma_{r}^{T}\left(\mathbf{R}_{r s}+\mathbf{Z}_{r} \mathbf{G} \mathbf{Z}_{s}^{T}\right) \mathbf{V}_{s s}^{-1},
$$

$\Sigma(\boldsymbol{\delta})=E(\boldsymbol{\delta}-\boldsymbol{\delta})(\boldsymbol{\delta}-\boldsymbol{\delta})^{T}, \mathrm{~B}_{\boldsymbol{\delta}}(\boldsymbol{\delta})$ is defined in the regularity condition (f) in the Appendix (Section 7).

Proof. The proof results directly (assuming our regularity conditions, our model and replacing $\mathbf{m}^{T} \mathbf{G} \mathbf{Z}_{s}^{T} \mathbf{V}_{s s}^{-1}$ by $\gamma_{r}^{T} \mathbf{V}_{r s} \mathbf{V}_{s s}^{-1}$ ) from the proof of the theorem of Datta and Lahiri (2000, 624) (called theorem A.2). The difference between Lemma 1 and the theorem presented in Żąło $(2009,110)$ results from the assumed model (including the number of blocks in the covariance matrix and the sizes of the matrices).

Remark 1. Under normality of $\mathbf{Y}$ the $g_{3}(\boldsymbol{\delta})$ given by Equation (20) approximates the difference between the MSE of EBLUP and the MSE of BLUP for the predictor considered by Royall (1976) (the predictor presented in Theorem 2) - see Żądło (2009, 107). Applying results presented by Żąłło (2009) to RC presented in the Appendix (Section 7), it can be proved that the order of approximation is $o\left(K^{-1}\right)$. It is worth noting that Equation (20) is the generalization of:

$$
g_{3}^{s}(\boldsymbol{\delta})=\operatorname{trace}\left(\frac{\partial \mathbf{b}^{\mathrm{T}}(\boldsymbol{\delta})}{\partial \boldsymbol{\delta}} \mathbf{V}_{s s}\left(\frac{\partial \mathbf{b}^{T}(\boldsymbol{\delta})}{\partial \boldsymbol{\delta}}\right)^{T} \Sigma(\boldsymbol{\delta})\right)
$$

where

$$
\mathbf{b}^{T}=\mathbf{m}^{T} \mathbf{G} \mathbf{Z}_{s}^{T} \mathbf{V}_{s s}^{-1},
$$

presented by Datta and Lahiri (2000), which approximates the difference between the MSE of EBLUP and the MSE of BLUP for the predictor considered by Henderson (1950) (the predictor presented in Theorem 1). Moreover, if we consider the problem of prediction of Equation (10), then Equation (22) is given by:

$$
\mathbf{b}^{T}=\gamma_{r}^{T} \mathbf{Z}_{r} \mathbf{G} \mathbf{Z}_{s}^{T} \mathbf{V}_{s s}^{-1} .
$$


Then, for models with $\mathrm{R}_{r s}=0$ we obtain

$$
g_{3}^{s}(\boldsymbol{\delta})=g_{3}(\boldsymbol{\delta}),
$$

where $g_{3}(\boldsymbol{\delta})=g_{3}^{s}(\boldsymbol{\delta})$, are given by Equations (21) and (20), respectively.

Lemma 2. Under Model 1, Model 2 and regularity conditions (RC) presented in Appendix (Section 7):

(i) $E_{*}\left(g_{1}\left(\boldsymbol{\delta}^{*}\right)\right)=g_{1}(\boldsymbol{\delta})+\mathbf{B}_{\boldsymbol{\delta}}^{T}(\boldsymbol{\delta}) \frac{\partial g_{1}(\boldsymbol{\delta})}{\partial \boldsymbol{\delta}}-g_{3}(\boldsymbol{\delta})+o_{p}\left(K^{-1}\right)$,

(ii) $E_{*}\left(g_{2}\left(\boldsymbol{\delta}^{*}\right)\right)=g_{2}(\boldsymbol{\delta})+o_{p}\left(K^{-1}\right)$,

(iii) $E_{*}\left(\hat{\theta}_{E B L U P}\left(\mathbf{Y}_{s}, \hat{\beta}\left(\boldsymbol{\delta}^{*}\right), \boldsymbol{\delta}^{*}\right)-\hat{\theta}_{E B L U P}\left(\mathbf{Y}_{s}, \hat{\beta}(\boldsymbol{\delta}), \boldsymbol{\delta}\right)\right)^{2}=g_{4}(\boldsymbol{\delta})+o_{p}\left(K^{-1}\right)$,

(iv) $E\left(g_{4}(\boldsymbol{\delta})\right)=g_{3}(\boldsymbol{\delta})+o\left(K^{-1}\right)$,

where $\left.g_{4}(\boldsymbol{\delta})=\operatorname{trace}\left(\frac{\partial c^{T}(\boldsymbol{\delta})}{\partial \boldsymbol{\delta}}\left(\mathbf{Y}_{s}-\mathbf{X}_{s} \hat{\beta}\right)\left(\mathbf{Y}_{s}-\mathbf{X}_{s} \hat{\beta}\right)^{T}\left(\frac{\partial c^{T}(\boldsymbol{\delta})}{\partial \boldsymbol{\delta}}\right)^{T} \Sigma(\boldsymbol{\delta})\right)\right)$.

Proof. Parts (i) and (ii) in Lemma 2 follow from (i) and (ii) in Lemma 1 (but under Model 2) using $E_{*}\left(\boldsymbol{\delta}^{*}-\boldsymbol{\delta}\right)=O_{p}\left(K^{-1}\right)$ (as Butar and Lahiri 2003, 74). The proof of (iii) we obtain using

$$
\begin{aligned}
& \hat{\theta}_{E B L U P}\left(\mathbf{Y}_{\mathrm{s}}, \hat{\beta}\left(\boldsymbol{\delta}^{*}\right), \boldsymbol{\delta}^{*}\right)-\hat{\theta}_{E B L U P}(\boldsymbol{\delta})= \\
& =\left(\boldsymbol{\delta}^{*}-\boldsymbol{\delta}\right) \frac{\partial c^{T}(\boldsymbol{\delta})}{\partial \boldsymbol{\delta}}\left(\mathbf{Y}_{s}-\mathbf{X}_{\mathrm{s}} \hat{\beta}\right)+O_{p^{*}}\left(K^{-1}\right)
\end{aligned}
$$

under Model 2, which results directly from Equation (25) in Żądło (2009, 108), which is the direct generalization of Equation (A.2) in Datta and Lahiri (2000, 623) used by Butar and Lahiri $(2003,74)$. The proof of part (iv) results from the RC, see Butar and Lahiri $(2003,75)$, which implies $\boldsymbol{\delta}-\boldsymbol{\delta}=o_{p}(1), \quad \Sigma(\boldsymbol{\delta})=O\left(K^{-1}\right), \quad \hat{\beta}(\boldsymbol{\delta})=\beta+o_{p}(1)$, $\Sigma(\boldsymbol{\delta})=\Sigma(\boldsymbol{\delta})+o_{p}\left(K^{-1}\right)$. Hence, $\frac{\partial c^{T}(\boldsymbol{\delta})}{\partial \boldsymbol{\delta}}=\frac{\partial c^{T}(\boldsymbol{\delta})}{\partial \boldsymbol{\delta}}+o_{p}(1)$ and:

$$
\begin{aligned}
& \frac{\partial c^{T}(\boldsymbol{\delta})}{\partial \boldsymbol{\delta}}\left(\mathbf{Y}_{s}-\mathbf{X}_{s} \hat{\beta}\right)\left(\mathbf{Y}_{s}-\mathbf{X}_{s} \hat{\beta}\right)^{T}\left(\frac{\partial c^{T}(\boldsymbol{\delta})}{\partial \boldsymbol{\delta}}\right)^{T} \Sigma(\boldsymbol{\delta})= \\
& =\frac{\partial c^{T}(\boldsymbol{\delta})}{\partial \boldsymbol{\delta}}\left(\mathbf{Y}_{s}-\mathbf{X}_{s} \beta\right)\left(\mathbf{Y}_{s}-\mathbf{X}_{s} \beta\right)^{T}\left(\frac{\partial c^{T}(\boldsymbol{\delta})}{\partial \boldsymbol{\delta}}\right)^{T} \Sigma(\boldsymbol{\delta})+ \\
& \quad+o_{p}\left(K^{-1}\right) .
\end{aligned}
$$

Finally, using the expressions for $g_{3}(\boldsymbol{\delta})$ and $g_{4}(\boldsymbol{\delta})$ we get the part (iv) of Lemma 2.

Theorem 3. Under Model 1, Model 2 and the RC, we have:

$$
E\left(\widehat{M S E}\left(\hat{\theta}_{E B L U P}\right)\right)-\operatorname{MSE}\left(\hat{\theta}_{E B L U P}\right)=o\left(K^{-1}\right)
$$

where $\widehat{M S E}\left(\hat{\theta}_{E B L U P}\right)$ is given by Equation (19). 
Proof. Using Lemma 1 and Lemma 2 we get:

$$
\begin{aligned}
& E\left(\widehat{M S E}\left(\hat{\theta}_{E B L U P}\right)\right)=E\left[g_{1}(\hat{\boldsymbol{\delta}})+g_{2}(\hat{\boldsymbol{\delta}})+\right. \\
- & E_{*}\left(g_{1}\left(\boldsymbol{\delta}^{*}\right)+g_{2}\left(\boldsymbol{\delta}^{*}\right)-\left(g_{1}(\hat{\boldsymbol{\delta}})+g_{2}(\hat{\boldsymbol{\delta}})\right)\right)+ \\
+ & E_{*}\left(\hat{\theta}_{E B L U P}\left(\mathrm{Y}_{s}, \hat{\boldsymbol{\beta}}\left(\boldsymbol{\delta}^{*}\right), \boldsymbol{\delta}^{*}\right)+\right. \\
- & \left.\left.\hat{\theta}_{E B L U P}\left(\mathrm{Y}_{s}, \hat{\beta}(\boldsymbol{\delta}), \boldsymbol{\delta}\right)\right)^{2}\right]= \\
= & E\left[g_{1}(\hat{\boldsymbol{\delta}})+g_{2}(\hat{\boldsymbol{\delta}})-g_{1}(\hat{\boldsymbol{\delta}})-\mathbf{B}_{\boldsymbol{\delta}}^{T}(\boldsymbol{\delta}) \frac{\partial g_{1}(\boldsymbol{\delta})}{\partial \boldsymbol{\delta}}+g_{3}(\hat{\boldsymbol{\delta}})+\right. \\
& \left.-g_{2}(\hat{\boldsymbol{\delta}})+g_{1}(\hat{\boldsymbol{\delta}})+g_{2}(\hat{\boldsymbol{\delta}})+g_{4}(\hat{\boldsymbol{\delta}})+\boldsymbol{o}_{p}\left(K^{-1}\right)\right] \\
= & E\left[g_{1}(\hat{\boldsymbol{\delta}})+g_{2}(\hat{\boldsymbol{\delta}})+g_{3}(\hat{\boldsymbol{\delta}})+g_{4}(\hat{\boldsymbol{\delta}})+\right. \\
& \left.-\mathbf{B}_{\boldsymbol{\delta}}^{T}(\boldsymbol{\delta}) \frac{\partial g_{1}(\boldsymbol{\delta})}{\partial \boldsymbol{\delta}}+\boldsymbol{o}_{p}\left(K^{-1}\right)\right]= \\
= & g_{1}(\boldsymbol{\delta})+g_{2}(\boldsymbol{\delta})+g_{3}(\boldsymbol{\delta})+o\left(K^{-1}\right) \\
= & M S E\left(\hat{\theta}_{E B L U P}\right)+o\left(K^{-1}\right),
\end{aligned}
$$

where the last equality in Equation (24) for the empirical version of Royall (1976)'s BLUP was proved by Żądło (2009, 110).

\section{Real Data Analyses}

We will show an application of the proposed method together with other MSE estimators for a real data set. To analyze statistical properties of our method, we will also present Monte Carlo simulation studies, taking the problem of model misspecification into account as well.

In the following analyses, prepared using $\mathrm{R}$ ( $\mathrm{R}$ Core Team 2019), we consider the real population data for $N=378$ Polish counties called poviats (NUTS 4 ) for $M=3$ periods for the years 2011-2013. Two poviats were excluded from the analysis - the first because of the lack of data, the second (Warsaw) as an outlier. Investments in companies (in hundreds of million PLN) and the number of new companies registered (in hundreds) are the variable of interest and the auxiliary variable, respectively. The data are divided into $D=28$ domains in the following way. Firstly, the population of poviats (NUTS 4) is divided into 16 voivodships (NUTS 2). Secondly, poviats in each voivodship are divided into two groups according to the type of poviat (city counties and land counties), but only if the sizes of both groups are at least 3. Domain sizes range from 3 to 37 (with the mean: 13.5). The problem of prediction of domain totals in the last period is considered.

In the first period, poviats are divided into two strata. The first stratum consists of poviats from domains that consist only of city counties. Other poviats belong to the second stratum. In the first period, a simple random sample without replacement is drawn from each stratum, (optimal allocation is used), in which the overall sample size is $n=38$. 
Sample sizes in domains range from 0 to 8 (with the mean: 1.36). In 9 domains zero sample sizes are observed. The same elements are in the samples in other periods (the balanced panel sample). This gives the division of the population in each period into the sample and the set of non-sampled elements.

\subsection{Application}

We mimic the real data analysis for the considered sample data set. We would like to find the model with the best goodness-of-fit measured by AIC and BIC criteria. Of course different measures of goodness-of-fit, including conditional AIC proposed by Vaida and Blanchard (2005) for clustered data, can be used as well. In the example, we do not group the profiles and hence the subscript $k$ is omitted. We study models with one auxiliary variable, with and without random effects, with and without constants that belong to the following two classes. Firstly,

$$
Y_{i j d}=\beta_{1} x_{i d j}+\beta_{2}+v_{1}+v_{2}+e_{i d j}
$$

where $i=1,2, \ldots, N ; d=1,2, \ldots, D, j=1,2, \ldots, M_{i d} ; \quad v_{1}, \quad v_{2}$ and $e_{i d j}$ are mutually independent, $v_{1} \sim N\left(0, \sigma_{1}^{2}\right), \quad v_{2} \sim N\left(0, \sigma_{2}^{2}\right)$ and $e_{i d j} \sim N\left(0, \sigma_{e}^{2}\right)$. Random effects $v_{1}$ and $v_{2}$ (where $v_{1} \neq v_{2}$ ) can be domain specific $\left(v_{d}\right)$, time specific $\left(v_{t}\right)$, domain-and-time specific $\left(v_{d t}\right)$ or profile specific $\left(v_{i d}\right)$. Secondly,

$$
Y_{i j d}=\left(\beta_{1}+v_{1}\right) x_{i d j}+\left(\beta_{2}+v_{2}\right) e_{i d j}
$$

where $i=1,2, \ldots, N ; \quad d=1,2, \ldots, D, j=1,2, \ldots, M_{i d}, \quad v_{1}, \quad v_{2}$ and $e_{i d j}$ are mutually independent, $v_{1} \sim N\left(0, \sigma_{1}^{2}\right), v_{2} \sim N\left(0, \sigma_{2}^{2}\right)$ and $e_{i d j} \sim N\left(0, \sigma_{e}^{2}\right)$. Random effects $v_{1}$ and $v_{2}$ (where $v_{1} \neq v_{2}$ or $v_{1}=v_{2}$ ) can be, for example, domain specific $\left(v_{d}\right)$, time specific $\left(v_{t}\right)$, domain-and-time specific $\left(v_{d t}\right)$ or profile specific $\left(v_{i d}\right)$.

The model with the smallest AIC and BIC criteria is given by

$$
Y_{i j d}=\left(\beta_{1}+v_{i d}\right) x_{i d j}+e_{i d j}
$$

REML estimates of model parameters are as follows: $\hat{\beta}_{1}=0.4194, \hat{\sigma}_{v}^{2}=$ 0.0778 and $\hat{\sigma}_{\mathrm{e}}^{2}=1.5194$. Based on the results of permutation tests we can claim that model parameters are statistically significant ( $\mathrm{p}$-values for tests of $\beta_{1}$ and $\hat{\sigma}_{v}^{2}$ are zero). The model belongs to the class of random regression coefficients models considered by Moura and Holt (1999) and Hobza and Morales (2013).

Remark 2. Firstly, in Model (25) the independence of random components $\left(e_{i d j}\right)$ is assumed, which means that $\mathbf{R}_{r s}=\mathbf{0}$ and hence, predictors (12a) and (14a) are identical and the equalities (16), (17) and (23) are true. Secondly, the model belongs to the class of mixed linear models with independent profile-specific random components, widely discussed by, for example, Verbeke and Molenbergh (2000). In this class of models, nonzero covariances between the variables of interest are observed only within profiles, and hence for the balanced panels samples we have $\mathbf{V}_{r s}=\mathbf{0}$ and (because $\mathbf{G}$ is diagonal) 
$\mathbf{Z}_{r} \mathbf{G} \mathbf{Z}_{s}^{T}=\mathbf{0}$. This implies that

$$
g_{3}(\boldsymbol{\delta})=g_{3}^{s}(\boldsymbol{\delta})=0,
$$

where $g_{3}(\boldsymbol{\delta})$ and $g_{3}^{s}(\boldsymbol{\delta})$ are given by Equations (21) and (20), respectively.

In Table 1 we present values for the following MSE estimators of the empirical version of the predictor $(14 \mathrm{a})$ :

- the MSE estimator based on the Taylor expansion proposed by Żąłło (2009), which is - for the considered model - equivalent to the MSE estimator of the empirical version of the predictor (12a) originally proposed by Datta and Lahiri (2000) (Taylor),

- the MSE estimator based on delete-one-profile jackknife applied for the empirical version of the predictor (14a), based on the idea presented by Jiang et al. (2002) (where delete-one-domain jackknife for empirical version of the predictor (7a) was studied) (jack),

- the MSE estimator based on delete-one-profile weighted-jackknife applied for the empirical version of the predictor (14a), based on the idea presented by Chen and Lahiri $(2002,2003)$ (where weighted delete-one-domain jackknife for the empirical version of the predictor (7a) was studied) ( $w$-jack),

- the parametric bootstrap MSE estimator studied by González-Manteiga et al. (2007, 2008) (boot1),

- the proposed parametric bootstrap MSE estimator given by (19) (boot2).

Jackknife and weighted jackknife MSE estimators are adapted for the considered predictor by: (i) in formulae presented by Jiang et al. $(2002,1,787)$ and Chen and Lahiri $(2002,474)$ replacing $g_{1}^{s}$ (.) and $g_{1}^{s}$ (.) (see Equations (7e) and (7f) with $g_{1}($.$) and g_{2}($.$) (see Equations$ (14c) and (14d)) and (ii) deleting profiles instead of domains in the case of estimation of $\boldsymbol{\delta}$ (in our model the number of blocks in $\mathbf{V}$ matrix is equal to the number of profiles).

In Table 1 we present results for all observed domain sample sizes. If the same sample size is observed in many domains, we present results only for two of them - with the smallest and the largest MSE estimates. For most of domains, the values of all MSE estimators except jack were very similar, which suggests similar stochastic properties (studied in the next section).

Table 1. Values of the MSE estimators for selected domains.

\begin{tabular}{lllrrrrr}
\hline$d$ & $n_{d}$ & $N_{d}$ & Taylor & jack & w-jack & boot1 & boot2 \\
\hline 13 & 0 & 14 & 36.4 & 32.2 & 36.4 & 36.7 & 36.5 \\
22 & 0 & 3 & 643.6 & 612.7 & 642.0 & 707.0 & 643.3 \\
12 & 1 & 4 & 8.1 & 7.7 & 8.0 & 7.8 & 8.1 \\
6 & 1 & 3 & 798.1 & 766.9 & 796.0 & 824.1 & 797.8 \\
27 & 2 & 4 & 87.0 & 83.4 & 86.8 & 85.4 & 87.0 \\
19 & 2 & 18 & 94.5 & 71.4 & 94.6 & 94.2 & 94.7 \\
11 & 3 & 21 & 85.6 & 65.4 & 85.7 & 89.6 & 85.8 \\
28 & 4 & 21 & 109.5 & 87.4 & 109.7 & 117.4 & 109.8 \\
17 & 6 & 31 & 326.1 & 242.9 & 326.6 & 335.2 & 326.9 \\
8 & 8 & 19 & 145.2 & 116.1 & 145.3 & 142.8 & 145.5 \\
\hline
\end{tabular}


All of the considered MSE estimators are based on the normal mixed model where the normality of random effects and random components is assumed, which is equivalent (logical biconditional) to normality Y (see Remark 3 ).

Remark 3. Assuming (3) (including independence of $\mathbf{v}$ and $\mathbf{e}$ and full column rank of $\mathbf{Z}), \mathbf{v}$ and $\mathbf{e}$ have multivariate normal distributions if and only if $\mathbf{Y}$ has the multivariate normal distribution. The above statement is true because both implications are true. The implication, that if $\mathbf{v}$ and $\mathbf{e}$ in Equation (3) have multivariate normal distributions, then $\mathbf{Y}$ has the multivariate normal distribution, is a standard result (see e.g., Muirhead (2005), 6 theorem 1.2.6 and 14 theorem 1.2.14). The implication, that if $\mathbf{Y}$ in (3) has the multivariate normal distribution, then $\mathbf{v}$ and $\mathbf{e}$ have multivariate normal distribution, results from the following two statements. Firstly, if $\mathbf{Y}$ in (3) has the multivariate normal distribution, then two random vectors of the same size $\mathbf{Z v}$ and $\mathbf{e}$ have multivariate normal distributions (see e.g., Muirhead (2005), 14 theorem 1.2.13). Secondly, if $\mathbf{Z v}$ has multivariate normal distribution and $\mathbf{Z}$ is of full rank, then $\mathbf{v}$ has the multivariate normal distribution. It is true, because there exists a linear transformation of $\mathbf{Z v}$ to $\mathbf{v}$ (e.g., $\left.\left(\left(\mathbf{Z}^{T} \mathbf{Z}\right)^{-1} \mathbf{Z}^{T}\right)(\mathbf{Z v})=\mathbf{v}\right)$ and then (e.g., Muirhead (2005), 6 theorem 1.2.6) normality of $\mathbf{Z v}$ implies normality of $\mathbf{v}$.
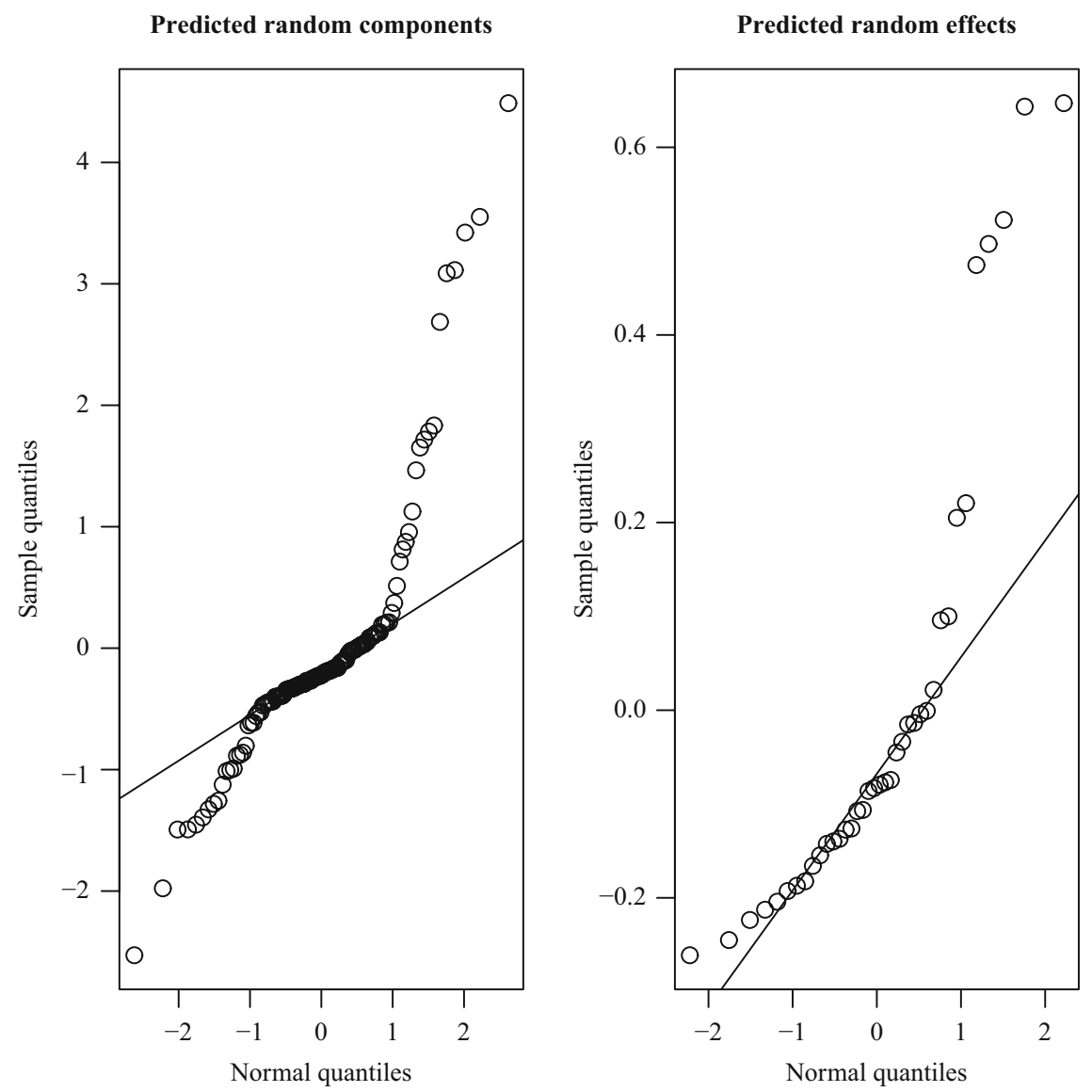

Fig. 1. Q-Q plots of predicted random components and random effects. 
The crucial point of the proofs of asymptotic unbiasedness of MSE estimators, where normality of $\mathbf{Y}$ (normality of random effects and random components) is required, is the approximate decomposition of the MSE of the EBLUP into: the MSE of the BLUP and the additional component usually denoted by $g_{3}($.$) (see Equation (20) for our case). The proof$ of the decomposition for the empirical version of the predictor studied by Henderson (1950) is considered by, for example, Kackar and Harville (1984, 855) and Robinson $(1991,19)$ and for the empirical version of the predictor studied by Royall (1976) and by Harville and Jeske (1992) in Section 2. Then, based on the decomposition (under normality of $\mathbf{Y}), g_{3}($.$) component is estimated using different methods and, finally, the$ MSE estimator is obtained. The assumption of normality of $\mathbf{Y}$ is used in the case of the MSE estimator based on Taylor's expansion by Datta and Lahiri $(2000,623)$ and Żądło (2009), for the weighted jackknife MSE estimator by Chen and Lahiri $(2003,908)$ and for the parametric bootstrap MSE estimators by Butar and Lahiri $(2003$, 66) and in our proposal (see Remark 1). In case of the jackknife MSE estimator the proof is different, but normality of $\mathbf{Y}$ is also required, as shown by Jiang et al. (2002, 1,803). For our data, the assumption of normality of $\mathbf{Y}$ (or equivalently the normality of $\mathbf{v}$ and $\mathbf{e}-$ see Remark 3 ) is not met - using the Shapiro test we checked normality using residuals $\mathbf{Y}_{s}-\mathbf{X}_{s} \hat{\beta}$ after the Cholesky transformation (p-value equals 0). Q-Q plots of predicted random effects $\hat{\mathrm{v}}=\tilde{\mathrm{v}}(\hat{\boldsymbol{\delta}})$ (where $\tilde{\mathrm{v}}(\boldsymbol{\delta})$ is given by Equation (7c)) and predicted random components (conditional residuals) $\hat{\mathrm{e}}_{s}=\mathbf{Y}_{s}-\mathbf{X}_{s} \hat{\beta}-\mathbf{Z}_{s} \hat{\mathbf{v}}$ are presented in Figure 1. Hence, in the next section we will study the biases and MSEs of the considered MSE estimators under normality and different non-normal cases. We are interested in comparing the behavior of our estimator with its competitors under different distributions.

\subsection{Simulation Study}

In the model-based simulation study prepared in R (R Core Team 2019), we analyze the same MSE estimators, the same data, the same model and the same division into the sampled and non-sampled sets as in the previous section. The number of iteration equals $L=5,000$.

Bootstrap MSE estimators are computed based on 200 replications.

The values of variable of interest are generated based on Model (25) (with parameters computed based on the whole population data), where random effects and random components are generated using the following distributions:

- normal,

- scaled t-Student with 3 degrees of freedom,

- shifted exponential,

- shifted log-normal (where the third standardized moment equals 3),

- shifted gamma (where the third standardized moment equals 4),

- shifted Pareto (where the third standardized moment equals 5).

Distributions with positive asymmetry are usually used in Monte Carlo simulation studies in economic applications (e.g., Białek 2014). The true MSEs are computed based on the following formula: $M S E=L^{-1} \sum_{l=1}^{L}\left(\hat{\theta}_{l}-\theta_{l}\right)^{2}$, where the number of Monte Carlo iterations equals $L=5000, \hat{\theta}_{l}$ and $\theta_{l}$ are computed for $l$ th iteration as Equations (14a) and (5a), respectively. 


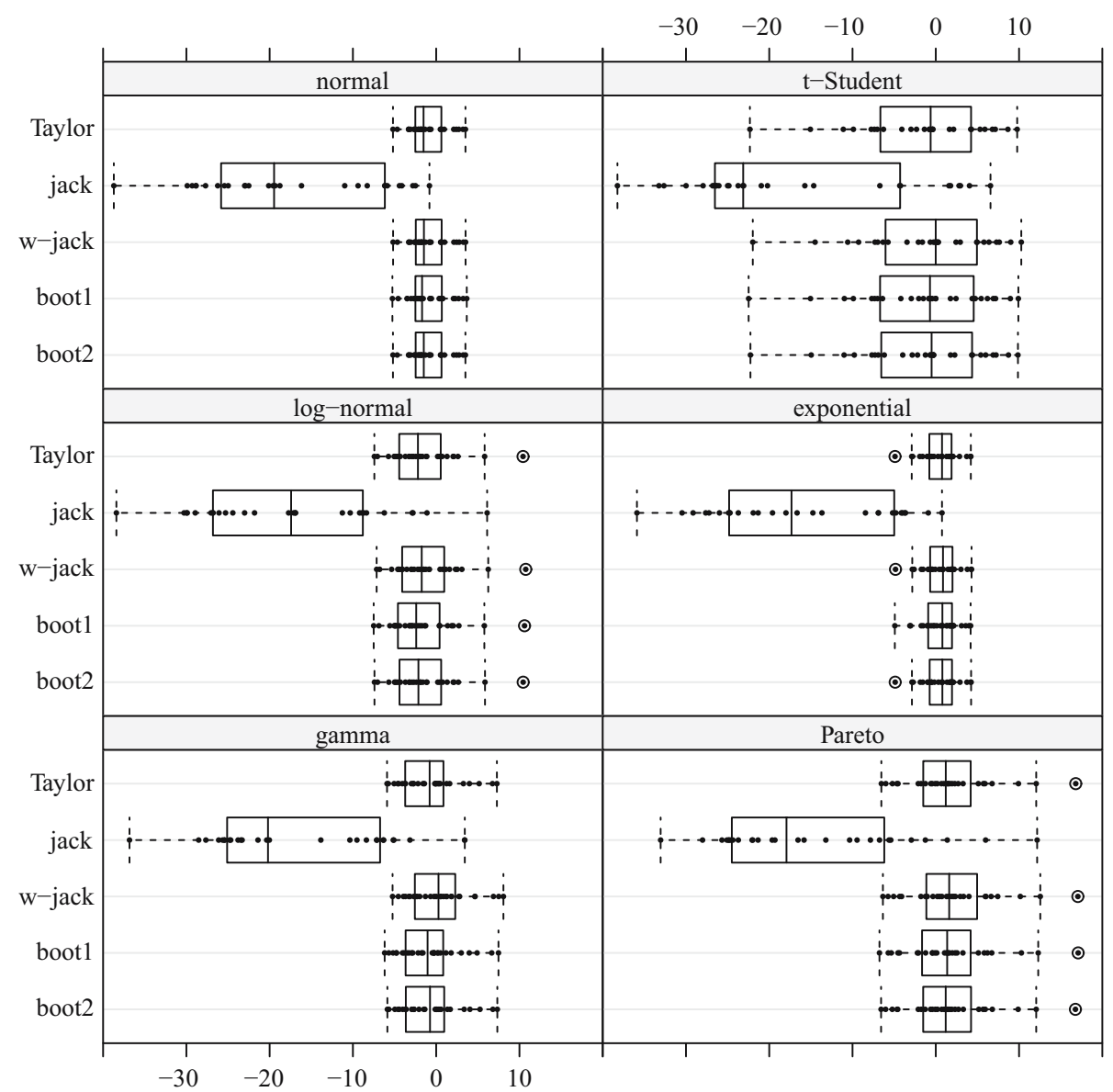

Fig. 2. Relative biases of MSE estimators for $D=28$ domains (in percent).

In Figure 2 we present relative biases of the MSE estimators computed as $r B(\widehat{M S E})=$ $M S E^{-1} L^{-1} \sum_{l=1}^{L}\left(\widehat{M S E}_{l}-M S E\right)$, where $\widehat{M S E}_{l}$ is the value of the MSE estimator computed for the $l$ th iteration. If the normality assumption is met, the relative biases of the estimators, except jack, are between approximately $-5 \%$ and $4 \%$. For other distributions, absolute biases are larger, but - in our opinion - acceptable for most of the domains. Moreover, median relative biases for all of the MSE estimators except jack are very close to zero.

Even though for non-normal distributions the increase of the absolute biases is in our opinion and this acceptable, we observe a large increase of relative RMSEs (see Figure 3) computed as $\operatorname{rRMSE}(\widehat{M S E})=M S E^{-1}\left(L^{-1} \sum_{l=1}^{L}\left(\widehat{M S E}_{l}-M S E\right)^{2}\right)^{0.5}$. Values of relative RMSEs for normal distribution are between approximately $18 \%$ and $29 \%$ (except jack), but for other distributions they even exceed $100 \%$ for some cases. It is interesting to note that although large absolute biases are observed for the jack MSE estimator (see Figure 2) it is the MSE estimator with the smallest RMSEs for non-normal distributions (see Figure 3).

Summing up the results of the Monte Carlo simulation study presented in the previous two paragraphs, we can state that all of the considered MSEs are robust on the lack of 


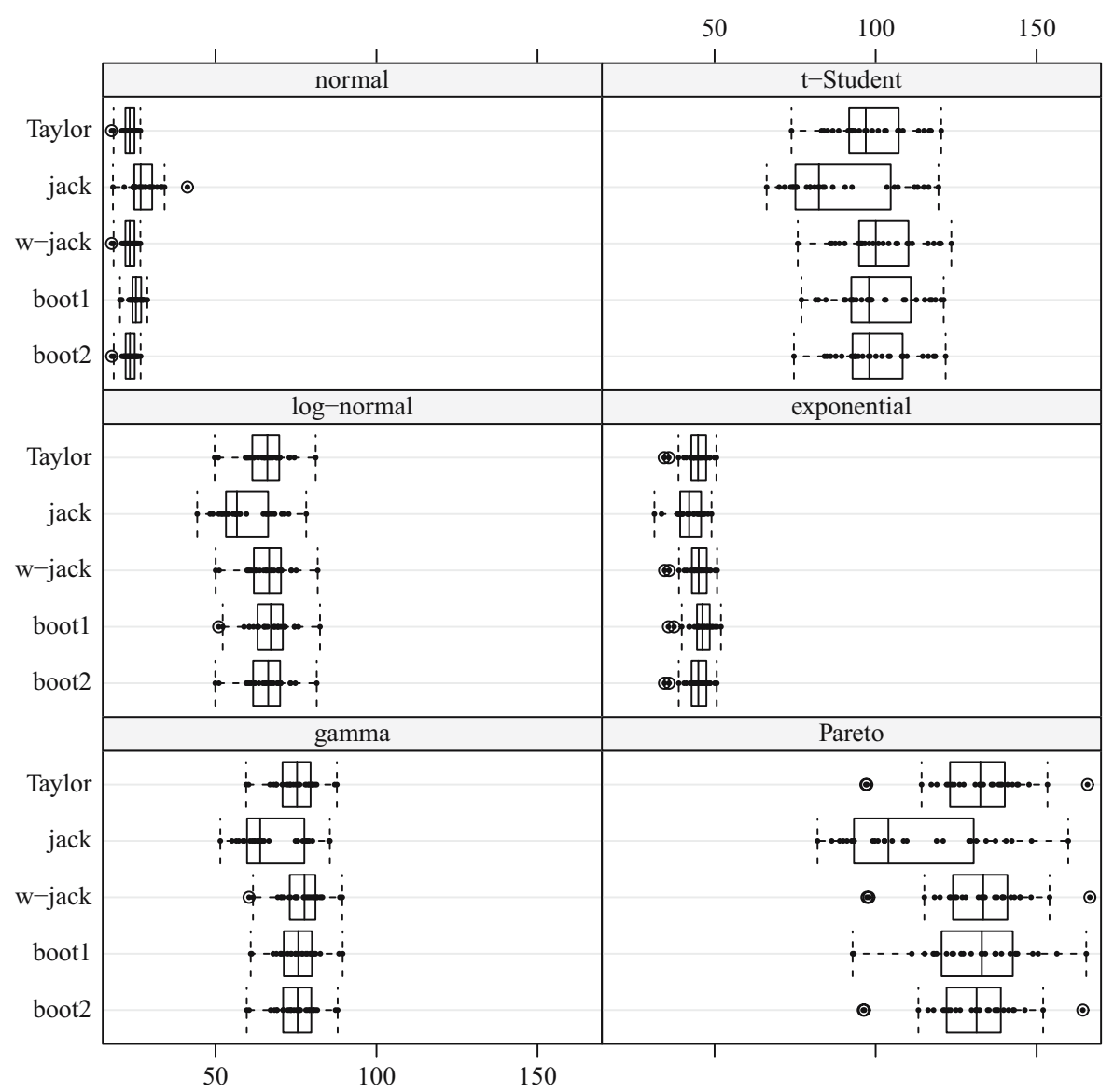

Fig. 3. Relative RMSEs of MSE estimators for $D=28$ domains (in percent).

normality taking into account their biases, but not if their MSEs are considered. We observe a large decrease of their accuracies both for the heavy-tailed scaled t-Student distribution and for positively skewed distributions, where for the stronger asymmetry a larger increase of the MSE is observed. Interesting results are observed for the jackknife MSE estimator for which slightly smaller MSEs are received in non-normal cases. Furthermore, the proposed parametric bootstrap MSE estimator, jackknife and weighted jackknife MSE estimators (that we adapted for the considered predictor) have very similar properties compared with the MSE estimator-based on the Taylor expansion. However, their advantage over the Taylor expansion-based MSE estimator is that they do not require derivation of the $g_{3}(\boldsymbol{\delta})$ component of the MSE, which can be problematic for complex superpopulation models.

\section{Conclusion}

In the article, we show that the empirical version of the best linear unbiased predictor proposed by Royall (1976) is a generalization of the empirical version of the predictor 
studied by Henderson (1950), together with the condition when they are equivalent. We generalize the parametric bootstrap MSE estimator proposed by Butar and Lahiri (2003) and prove its asymptotic unbiasedness. The proof of asymptotic unbiasedness requires normality of random effects and random components, which is a limitation of the method but is also a typical assumption for other MSE estimators. This is the reason why, in the application and in the simulation study, the properties of the proposed MSE estimator are compared with other MSE estimators, showing that they have very similar properties both under normality and non-normal cases. However, our method has the advantage that it does not require derivation of the $g_{3}($.$) component of the MSE, as the MSE estimator is$ based on Taylor's expansion, which can be crucial for models with a complex covariance structure.

\section{Appendix}

We assume the following regularity conditions, which will be referred to as (RC). The following regularity conditions (a), (b), (e), (f), (g) are direct generalizations of the respective regularity conditions proposed by Butar and Lahiri (2003) for the proposed longitudinal model (i.e., the definition sizes of the matrices cover the case of longitudinal data and the assumption that population and subpopulations can change in time). We replace regularity conditions (c) and (d) proposed by Butar and Lahiri (2003) with the following regularity conditions (c) and (d) to cover both the more general predictor (the empirical version of the predictor proposed by Royall 1976) and the proposed model. The regularity conditions $(\mathrm{RC})$ are:

(a) The elements of matrices $\mathbf{X}_{k}$ and $\mathbf{Z}_{k}$ are uniformly bounded such that $\left\{\mathbf{X}_{s}^{T} \mathbf{V}_{s s}^{-1} \mathbf{X}_{s}\right\}=$ $[O(K)]_{p \times p}$

(b) $\sup _{1 \leq d \leq D} n_{k}<\infty$ and $\sup _{1 \leq d \leq D} r_{k}<\infty$,

(c) $\mathbf{X}_{r}^{T} \gamma_{r}-\mathbf{X}_{s}^{T} \mathbf{V}_{s s}^{-1} \mathbf{V}_{s r} \gamma_{r}=[O(1)]_{p \times 1}$,

(d) $\frac{\partial}{\partial \boldsymbol{\delta}_{c}} \mathbf{X}_{s}^{T} \mathbf{V}_{s s}^{-1} \mathrm{~V}_{s r} \gamma_{r}=[O(1)]_{p \times 1}$ where $c=1,2, \ldots, q$,

(e) $\mathbf{R}_{s k}(\boldsymbol{\delta})=\sum_{c=0}^{q} \boldsymbol{\delta}_{j} \mathbf{C}_{k c} \mathbf{C}_{k c}^{T}$ and $\mathbf{G}_{k}(\boldsymbol{\delta})=\sum_{c=0}^{q} \boldsymbol{\delta}_{j} \mathbf{F}_{k c} \mathbf{F}_{k c}^{T}$ where $\boldsymbol{\delta}_{0}=1, \mathbf{C}_{k c}$ and $\mathbf{F}_{k c}$ $(k=1,2, \ldots, K ; c=0,1, \ldots, q)$ are known matrices of the order $n_{k} \times r_{k}$ and $r_{k} \times r_{k}$, respectively, and the elements are uniformly bounded known constants such that $\mathbf{R}_{s k}(\boldsymbol{\delta})$ and $\mathbf{G}_{k}(\delta)(k=1,2, \ldots, K)$ are all positive definite matrices. In special cases, some of $\mathbf{C}_{k c}$ and $\mathbf{F}_{k c}$ may be null matrices.

(f) $\hat{\boldsymbol{\delta}}$ is an estimator of $\boldsymbol{\delta}$ that satisfies (i) $\hat{\boldsymbol{\delta}}-\boldsymbol{\delta}=O_{p}\left(K^{-1 / 2}\right)$, (ii) $\hat{\boldsymbol{\delta}}-\hat{\boldsymbol{\delta}}_{M L}=O_{p}\left(K^{-1}\right)$ (where $\hat{\boldsymbol{\delta}}_{M L}$ is the maximum-likelihood estimator of $\boldsymbol{\delta}$ ), (iii) $\hat{\boldsymbol{\delta}}\left(\mathbf{Y}_{s}\right)=\hat{\boldsymbol{\delta}}\left(-\mathbf{Y}_{s}\right.$ ), (iv) $\hat{\boldsymbol{\delta}}\left(\mathbf{Y}_{s}+\mathbf{X}_{s} \mathrm{~b}\right)=\hat{\boldsymbol{\delta}}\left(\mathbf{Y}_{s}\right)$, for any $\mathrm{b} \in R^{p}$ and for all $\mathbf{Y}_{s}$. Assume that $E(\hat{\boldsymbol{\delta}}-\boldsymbol{\delta})=$ $\mathrm{B}_{\boldsymbol{\delta}}(\boldsymbol{\delta})+o\left(K^{-1}\right)$, which means that the approximate (to the order $o\left(K^{-1}\right)$ ) formula of the bias of $\boldsymbol{\delta}$ is known.

$(\mathrm{g}) E(\hat{\beta}(\boldsymbol{\delta})-\beta)(\boldsymbol{\delta}-\boldsymbol{\delta})^{T}=o\left(K^{-1}\right)$.

\section{References}

Białek, J. 2014. "Simulation study of an original price index formula." Communications in

Statistics - Simulation and Computation 43: 285-297. DOI: http://doi.org/10.1080/ 03610918.2012.700367. 
Butar, F.B. and P. Lahiri. 2003. "On measures of uncertainty of empirical Bayes smallarea estimators.” Journal of Statistical Planning and Inference 112: 63-76. DOI: http:// doi.org/10.1016/S0378-3758(02)00323-3.

Chen, S. and P. Lahiri, 2002. "A weighted jackknife MSPE estimator in small-area estimation." In Proceedings of the Section on Survey Research Methods: American Statistical Association, May 14-19, 2002: 473-477. Florida, VAL: American Statistical Association. Available at: http://www.asasrms.org/Proceedings/y2002/Files/ JSM2002-001127.pdf (accessed January 2020).

Chen, S. and P. Lahiri. 2003. "A comparison of different MSPE estimators of EBLUP for the Fay-Herriot model." In Proceedings of the Section on Survey Research Methods: American Statistical Association, May 15-18, 2003: 905-911. Nashville, VAL: American Statistical Association. Available at: http://www.asasrms.org/Proceedings/ y2003/Files/JSM2003-000585.pdf (accessed January 2020).

D’Aló, M., S. Falorsi, and F. Solari. 2017. "Space-Time Unit-Level EBLUP for Large Data Sets." Journal of Official Statistics 33: 61-77. DOI: http://doi.org/10.1515/ jos-2017-0004.

Das, K., J. Jiang, and J.N.K. Rao. 2004. "Mean squared error of empirical predictor.” The Annals of Statistics 32: 818-840. DOI: http://doi.org/10.1214/009053604000000201.

Datta, G.S. and P. Lahiri. 2000. "A unified measure of uncertainty of estimated best linear unbiased predictors in small area estimation problems." Statistica Sinica 10: 613-627. Available at: http://www3.stat.sinica.edu.tw/statistica/oldpdf/A10n214.pdf (accessed January 2020).

Fabrizi, E., M.R. Ferrante, and S. Pacei. 2007. "Small area estimation of average household income based on unit level models for panel data." Survey Methodology 33: 187-198. Available at: https://www150.statcan.gc.ca/n1/en/pub/12-001-x/2007002/ article/10496-eng.pdf?st=eifh-iS (accessed January 2020).

González-Manteiga, W., M.J. Lombardía, I. Molina, D. Morales, and L. Santamaría. 2007. "Estimation of the mean squared error of predictors of small area linear parameters under a logistic mixed model." Computational Statistics and Data Analysis 51: 2720-2733. DOI: http://doi.org/10.1016/j.csda.2006.01.012.

González-Manteiga, W., M.J. Lombardía, I. Molina, D. Morales, and L. Santamaría. 2008. "Bootstrap mean squared error of small-area EBLUP." Journal of Statistical Computation and Simulation 78: 443-462. DOI: http://doi.org/10.1080/ 00949650601141811.

Harville, D.A. and D.R. Jeske. 1992. "Mean square error of estimation or prediction under general linear model." Journal of Statistical Computation and Simulation 87: 724-731. DOI: http://doi.org/10.1080/01621459.1992.10475274.

Henderson, C.R. 1950. "Estimation of genetic parameters (Abstract)." Annals of Mathematical Statistics 21: 309-310.

Hobza, T. and D. Morales. 2013. "Small area estimation under random regression coefficient models." Journal of Statistical Computation and Simulation 83: 2160-2177. DOI: http://doi.org/10.1080/00949655.2012.684094.

Hobza, T., D. Morales, and L. Santamaría. 2018. "Small area estimation of poverty proportions under unit-level temporal binomial-logit mixed models." TEST 27: 270-294. DOI: http://doi.org/10.1007/s11749-017-0545-3. 
Jiang, J. 1996. "REML Estimation: Asymptotic Behavior and Related Topics." The Annals of Statistics 24: 255-286. DOI: http://doi.org/10.1214/aos/1033066209.

Jiang, J. and P. Lahiri. 2006. "Mixed model prediction and small area estimation." Test 15: 1-96. DOI: http://doi.org/10.1007/BF02595419.

Jiang, J., P. Lahiri, and S.-M. Wan. 2002. "Unified jackknife theory for empirical best prediction with M-estimation.” The Annals of Statistics 30: 1782-1810. DOI: http://doi. org/10.1214/aos/1043351257.

Kackar, R.N. and D.A. Harville. 1981. "Unbiasedness of two-stage estimation and prediction procedures for mixed linear models." Communications in Statistics, Ser. A 10: 1249-1261. DOI: http://doi.org/10.1080/03610928108828108.

Kackar, R.N. and D.A. Harville. 1984. "Approximations for standard errors of estimators of fixed and random effects in mixed linear models." Journal of the American Statistical Association 79: 853-862. DOI: http://doi.org/10.1080/01621459.1984.10477102.

Marhuenda, Y., I. Molina, and D. Morales. 2013. "Small area estimation with spatiotemporal Fay-Herriot models." Computational Statistics \& Data Analysis 58: 308-325. DOI: http://doi.org/10.1016/j.csda.2012.09.002.

Molina, I., D. Morales, M. Pratesi, and N. Tzavidis. eds. 2010. "Final small area estimation developments and simulation results." SAMPLE project: Small Area Methods for Poverty and Living Conditions Estimates. http://www.sample-project.eu/images/ stories/docs/samplewp2d12\%2616_saefinal.pdf (accessed January 2020).

Moura, F.A.S. and D. Holt. 1999. "Small area estimation using multilevel models." Survey Methodology 25: 73-80.

Morales, D. and L. Santamaría. 2019. "Small area estimation under unit-level temporal linear mixed models." Journal of Statistical Computation and Simulation. 89: 1592-1620. DOI: http://doi.org/10.1080/00949655.2019.1590578.

Muirhead, R.J. 2005. Aspects of Multivariate Statistical Theory. New Jersey: John Wiley \& Sons.

Nissinen, K. 2009. Small area estimation with linear mixed models for unit-level panel and rotating panel data. Dissertation, University of Jyväskylä.

Pagliarella, M.C. and R. Salvatore. 2016. "Unit Level Spatio-temporal Models." In Analysis of Poverty Data by Small Area Estimation, edited by M. Pratesi, 227-243. Chichester: Wiley.

Prasad, N.G.N. and J.N.K. Rao. 1990. "The estimation of mean the mean squared error of small-area-estimators." Journal of the American Statistical Association 85: 163-171. DOI: http://doi.org/10.1080/01621459.1990.10475320.

$\mathrm{R}$ Core Team. 2019. R: A language and environment for statistical computing. $\mathrm{R}$ Foundation for Statistical Computing, Vienna. Available at: https://www.R-project.org/ (accessed January 2020).

Rao, J.N.K. and I. Molina. 2015. Small area estimation. Second edition. New Jersey: Wiley.

Rao, J.N.K. and M. Yu. 1994. "Small area estimation by combining time-series and crosssectional data." Canadian Journal of Statistics 22: 511-528. DOI: http://doi.org/10. $2307 / 3315407$.

Robinson, G.K. 1991. "That BLUP is a good thing: the estimation of random effects." Statistical Science 6: 15-51. DOI: http://doi.org/10.1214/ss/1177011926. 
Royall, R.M. 1976. "The linear least squares prediction approach to two-stage sampling." Journal of the American Statistical Association 71: 657-473. DOI: http://doi.org/10. 1080/01621459.1976.10481542.

Saei, A. and R. Chambers. 2003. Small Area Estimation Under Linear and Generalized Linear Mixed Models with Time and Area Effects. S3RI, Methodology Working Paper M03/15r, Southampton: University of Southampton. Available at: https://eprints.soton. ac.uk/8165/1/8165-01.pdf (accessed January 2020).

Schmid, T. and R. Münnich. 2014. "Spatial robust small area estimation." Statistical Papers 55: 653-670. DOI: http://doi.org/10.1007/s00362-013-0517-y.

Stukel, D.M. and J.N.K. Rao. 1999. "On small-area estimation under two-fold nested error regression models.” Journal of Statistical Planning and Inference 78: 131-147. DOI: http://doi.org/10.1016/S0378-3758(98)00211-0.

Vaida, F. and S. Blanchard. 2005. "Conditional Akaike Information for Mixed-Effects Models.” Biometrika 92: 351-370. DOI: http://doi.org/10.1093/biomet/92.2.351.

Valliant, R., A.H. Dorfman, and R.M. Royall. 2000. Finite population sampling and inference. A prediction approach. New York: John Wiley \& Sons.

Verbeke, G. and G. Molenberghs. 2000. Linear mixed models for longitudinal data. New York: Springer-Verlag.

Żądło, T. 2004. "On unbiasedness of some EBLU predictor." In Proceedings in Computational Statistics 2004, Physica-Verlag, Heidelberg-New York, August 23-27, 2004. 2019-2026. Prague, VAL: International Association for Statistical Computing. Available at: https://link.springer.com/book/10.1007/978-3-7908-2656-2 (accessed January 2020).

Żądło, T. 2009. "On MSE of EBLUP.” Statistical Papers 50: 101-118. DOI: http://doi. org/10.1007/s00362-007-0066-3.

Żąłło, T. 2014. "On the Prediction of the Subpopulation Total Based on Spatially Correlated Longitudinal Data." Mathematical Population Studies 21: 30-44. DOI: http://doi.org/10.1080/08898480.2013.836387.

Żądło, T. 2015a. "On longitudinal moving average model for prediction of subpopulation total." Statistical Papers 56: 749-771. DOI: http://doi.org/10.1007/s00362-0140607-5.

Żądło, T. 2015b. "On prediction for correlated domains in longitudinal surveys." Communications in Statistics - Theory and Methods 44: 683-697. DOI: http://doi.org/10.1080/ 03610926.2013 .857867$.

Received April 2019

Revised October 2019

Accepted January 2020 